\title{
Rôle de la cystoscopie de fluorescence en lumière bleve induite par Hexvix dans la prise en charge des tumeurs urothéliales vésicales
}

\author{
Adil Ait Sakel, MD; ${ }^{*}$ Adil Mazdar, MD; ${ }^{\dagger}$ Faustin N'guibou, MD* \\ ‘Service d'urologie, Hôpital d'Auxerre, France ; †'Service d'urologie A, Rabat, Morocco
}

Cite as: Can Urol Assoc J 2014;8(3-4):e216-20. http://dx.doi.org/10.5489/cuai.677 Published online March 11, 2014.

\section{Résumé}

Objectifs : Évaluer la faisabilité, la fiabilité, le rôle actuel et l'intérêt de la cystoscopie de fluorescence en lumière bleue induite par I'hexaminolévulinate (Hexvix, Photocure ASA) pour la détection des tumeurs urothéliales. Préciser le profil des tumeurs plus particulièrement concernées par cette technique.

Patients et méthodes : De janvier 2009 à janvier 2010, une lésion vésicale papillaire typique ou une lésion suspecte a été décelée en cystoscopie chez un total de 30 patients (64 prélèvements). Une résection endoscopique a été réalisée après la détection des lésions vésicales en lumière blanche et en lumière bleue (Hexvix). Une limite de l'étude est l'utilisation répétée d'Hexvix aux fins du suivi de patients porteurs d'un cancer de la vessie n'a pas été étudiée. Résultats : L'âge moyen des patients était de 70 ans (56-84 ans ; 27 hommes et 3 femmes). La cytologie préopératoire s'est révélée positive chez 17 patients, et négative chez 13 patients. Notre étude de 30 patients a permis d'analyser 64 prélèvements. Trentecinq tumeurs papillaires et 10 lésions non papillaires, mais suspectes, ont été repérées en lumière blanche. Cinquante-six prélèvements présentaient une fluorescence positive en lumière bleue, dont 19 lésions étaient seulement décelables sous cette source de lumière.

Conclusion : Notre étude nous a permis de conclure que la cystoscopie de fluorescence à l'hexaminolévulinate améliore sensiblement la détection des tumeurs superficielles de la vessie, particulièrement des carcinomes in situ (CIS). De plus, elle permet une résection plus complète qu'en cystoscopie standard à la lumière blanche et diminue le taux de tumeurs résiduelles après résection transurétrale de la vessie (RTUV).

\section{Introduction}

Chaque année, 2,7 millions de patients reçoivent un traitement pour un carcinome vésical. Dans la majorité des cas, les tumeurs urothéliales apparaissent après l'âge de 60 ans $\mathrm{s}^{1,2}$. En France, cette pathologie occupe la septième place, tous cancers confondus (INVSS 2008), et constitue le deuxième cancer urologique en importance après celui de la prostate. Le carcinome vésical est responsable de $3 \%$ des décès par cancer. Son incidence est en augmentation d'environ $1 \%$ par an, mais le taux de mortalité qui lui est associé semble diminuer chez I'homme ${ }^{3}$.

La détection précoce des tumeurs de la vessie est essentielle pour améliorer la prise en charge thérapeutique et le pronostic de cette maladie, qui restent liés au stade et au grade tumoral initial. En effet, lors du diagnostic initial, de $75 \%$ à $85 \%$ des tumeurs sont des tumeurs de la vessie n'infiltrant pas le muscle (TVNIM). De ce nombre, de $60 \%$ à $70 \%$ récidiveront la première année, et de $10 \%$ à 20 $\%$ se mueront en tumeurs invasives ou métastatiques (ou les deux). La fréquence de ces récidives est probablement liée au fait qu'il persiste, après résection endoscopique, des lésions urothéliales non diagnostiquées (lésions dysplasiques planes ou de CIS, tumeurs papillaires de très petite taille). Certaines séries évaluent le taux de ces tumeurs résiduelles à près de $70 \%{ }^{4}$.

La présence d'un CIS vésical a d'ailleurs été clairement associée à un risque de récidive et de progression plus important, lequel est responsable de coûts de traitement supplémentaires. La principale lacune de la cystoscopie conventionnelle en lumière blanche (LB) est sa faible capacité de détection des lésions planes telles que le CIS ou les dysplasies. De plus, la cystoscopie en LB peut être mise en défaut dans certains cas de lésions papillaires, en particulier quand elles sont petites et multiples ${ }^{5}$.

Le principe de la cystoscopie de fluorescence (technique Hexvix, Photocure ASA) repose sur l'accumulation de protoporphyrine IX dans les tissus tumoraux après une instillation d'acide 5-aminolévulinique (5-ALA) ou son ester, I'hexaminolévulinate $(\mathrm{HAL})^{6,7}$. La protoporphyrine IX émet une lumière intense sous lumière bleue. La cystoscopie de fluorescence augmente ainsi le contraste visuel entre les tissus bénins et malins dans la vessie ${ }^{3}$.

Dans une étude prospective, nous avons voulu évaluer I'incidence de la technique Hexvix sur la fréquence de 
détection des tumeurs urothéliales et préciser le profil des tumeurs plus particulièrement concernées par le ciblage par Hexvix.

\section{Limites de notre étude}

L'utilisation répétée d'Hexvix aux fins du suivi de patients porteurs d'un cancer de la vessie n'a pas été étudiée. En effet, les séries consacrées aux RTUV de tumeurs initiales et aux RTUV de tumeurs récidivées font défaut dans la littérature actuelle, les études publiées regroupant dans une même cohorte tumeurs initiales et récidivantes.

\section{Patients et méthodes}

De janvier 2009 à janvier 2010, un total de 30 patients présentant une lésion vésicale papillaire typique ou une lésion suspecte, décelée en cystoscopie, ont été inclus dans cette étude prospective. Tous les patients ont été informés de l'utilisation de la technique Hexvix et ont donné leur consentement. Les règles de bonnes pratiques et les recommandations du laboratoire ont été respectées.

\section{Méthodologie}

Une solution d'HAL (50 ml, 1,7 mg/ml, GE Healthcare) dans une solution tampon a été instillée dans la vessie au moyen d'une sonde vésicale, 60 minutes avant la résection transurétrale de la vessie (RTUV). Après vidange vésicale et lavage vésical par gravité, une inspection à la lumière blanche a été réalisée. Toutes les lésions ont été qualifiées (tumeurs papillaires, zones suspectes) et localisées sur une cartographie vésicale (fig. 1). Pendant la période opératoire, une procédure identique a ensuite été réalisée en lumière bleue avec une source lumineuse au xénon. La RTUV a finalement été effectuée, emportant toutes les lésions décelées en lumière blanche et en lumière bleue (fig. 2).

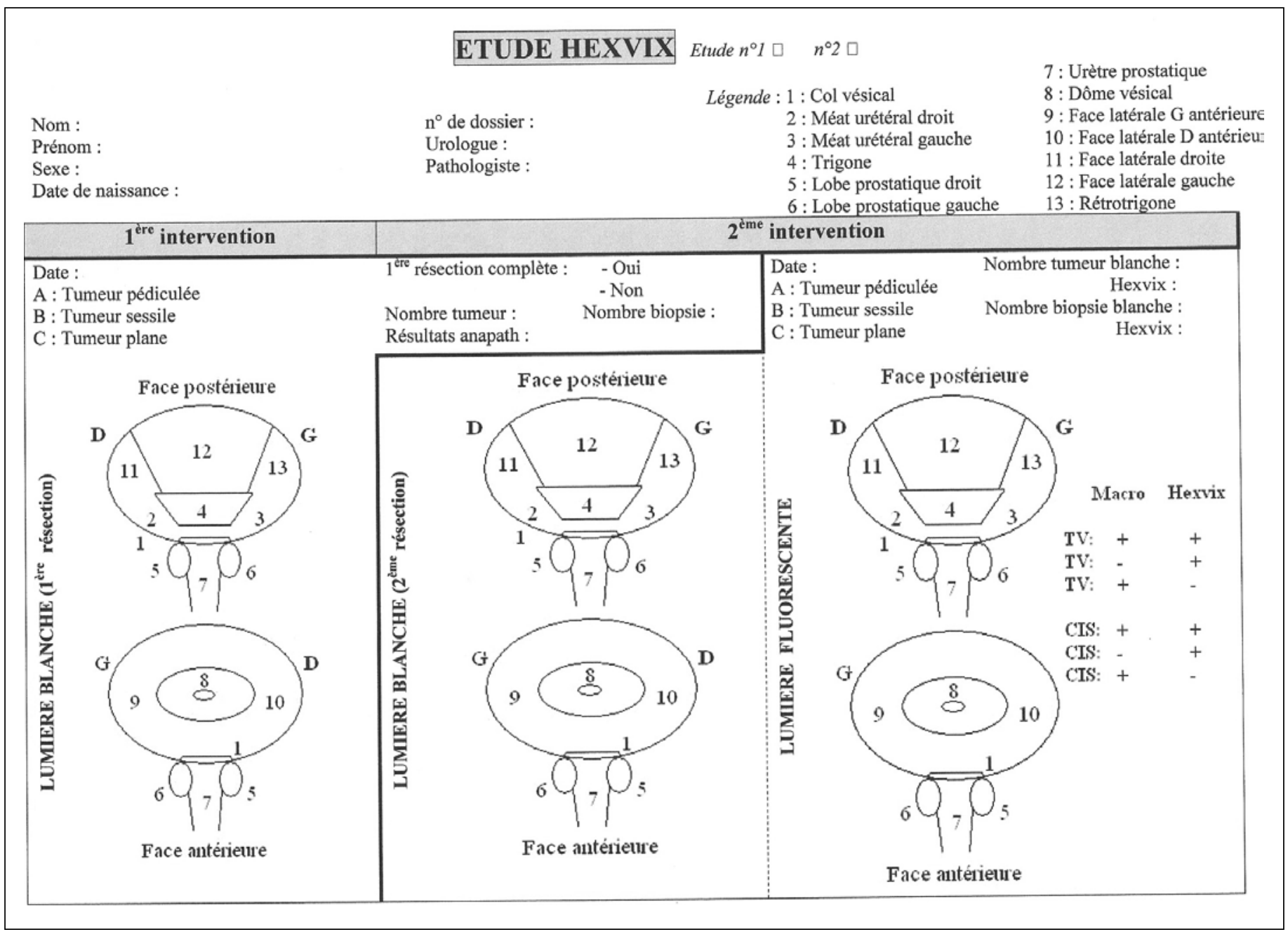

Fig. 1. Fiche patient - étude Hexvix. 


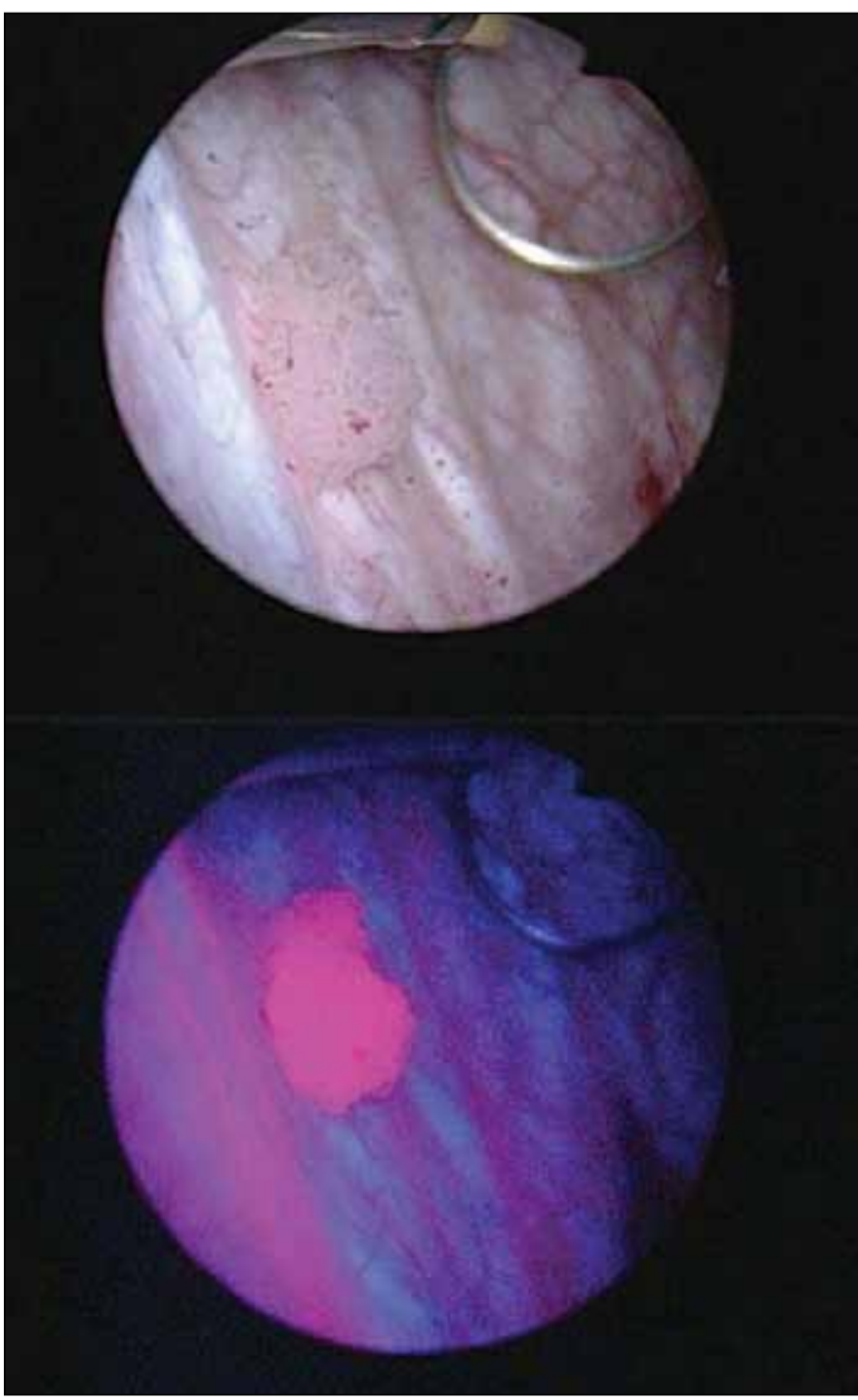

Fig. 2. Aspect en lumière blanche et en lumière bleue d'une tumeur urothéliale pTa de faible grade.

\section{Résultats}

Notre étude a porté sur 30 patients ( 27 hommes et 3 femmes) dont l'âge moyen était de 70 ans (56-84). L'étude de ces 30 patients a permis d'effectuer l'analyse de 64 prélèvements ( 2 prélèvements en moyenne par patient) (fig. 3 ).

Trente-cinq tumeurs papillaires et 10 zones non papillaires, mais suspectes (possibilité de dysplasie ou de carcinome in situ [CIS]), ont été décelées en lumière blanche. Cinquante-six prélèvements avaient une fluorescence positive en lumière bleue. Parmi les 35 tumeurs d'aspect papillaire en lumière blanche, 30 tumeurs $(85,7 \%)$ ont été confirmées à l'examen pathologique et 5 fragments $(14,2$ $\%)$ ont été considérés comme des lésions de dysplasie avec fluorescence positive. Parmi les 10 prélèvements considérés comme suspects en lumière blanche, aucun n'était tumoral.
La fréquence des faux positifs en lumière blanche et en lumière bleue était équivalente $(14,2 \%)$ pour les tumeurs $\mathrm{d}$ 'aspect papillaire. Pour les zones suspectes en lumière blanche, 2 zones sur 10 (20 \%) étaient positives en lumière bleue. Aucun de ces 10 prélèvements ne s'est avéré tumoral.

Dans cette étude, 39 tumeurs ont été diagnostiquées en lumière bleue, soit 9 localisations tumorales supplémentaires (9CIS). Parmi les 21 prélèvements effectués avec ou pour une fluorescence positive, 12 prélèvements ne mettaient pas en évidence de localisation tumorale lors de l'examen pathologique $(57,2 \%$ de faux positifs), contre 30 sur 45 $(66,6 \%)$ en lumière blanche seulement, et 19 prélèvements $(29,6 \%)$ ont été réalisés pour une fluorescence positive à I'Hexvix seulement (fig. 3).

\section{Discussion}

La RTUV est l'une des procédures les plus fréquentes pour I'urologue, et constitue la base du diagnostic et du traitement des tumeurs superficielles de la vessie. Cependant, la principale lacune de la cystoscopie conventionnelle en LB est sa faible capacité de détection des lésions planes telles que le CIS ou les dysplasies. De plus, la cystoscopie en lumière blanche peut être mise en défaut dans certains cas de lésions papillaires, en particulier quand elles sont petites et multiples ${ }^{5}$.

Le principe de la cystoscopie de fluorescence repose sur I'accumulation de protoporphyrine IX dans les tissus tumoraux après une instillation $\mathrm{d}^{\prime}$ acide 5 -ALA ou de son ester $\mathrm{HAL}^{6,7}$. La protoporphyrine IX émet une lumière intense sous lumière bleue. La cystoscopie de fluorescence augmente ainsi le contraste visuel entre les tissus bénins et malins dans la vessie ${ }^{6}$.

En conséquence, la détection des lésions planes de CIS échappe fréquemment à la cystoscopie standard, et la récidive précoce d'une tumeur de la vessie dès la première cystoscopie de contrôle témoigne parfois du caractère incomplet de la résection initiale. Le but de la fluorescence est de rendre " visible » ce qui échappe à la cystoscopie standard. Dans plus de $50 \%$ des cas, la cystoscopie en fluorescence (en lumière bleue) a démontré sa supériorité pour ce qui est de la détection des tumeurs de la vessie superficielles, et tout particulièrement du CIS, par rapport à la cystoscopie standard en $\mathrm{LB}^{8-11}$.

Dans notre série, nous confirmons ce fait : près de $13 \%$ de tumeurs supplémentaires ont été diagnostiquées, dont tous les CIS. Cette amélioration du diagnostic s'est révélée cependant moins importante que dans les autres études publiées. En effet, trois études de phase $1 \mathrm{II}^{8,9,12}$ ont été menées au cours des dernières années. Ces trois études de phase III font état d'un taux de détection des lésions de $93 \%$ à $97 \%$ pour la cystoscopie à fluorescence, alors qu'elle est de l'ordre de $77 \%$ à $79 \%$ pour la cystoscopie en lumière blanche. En 


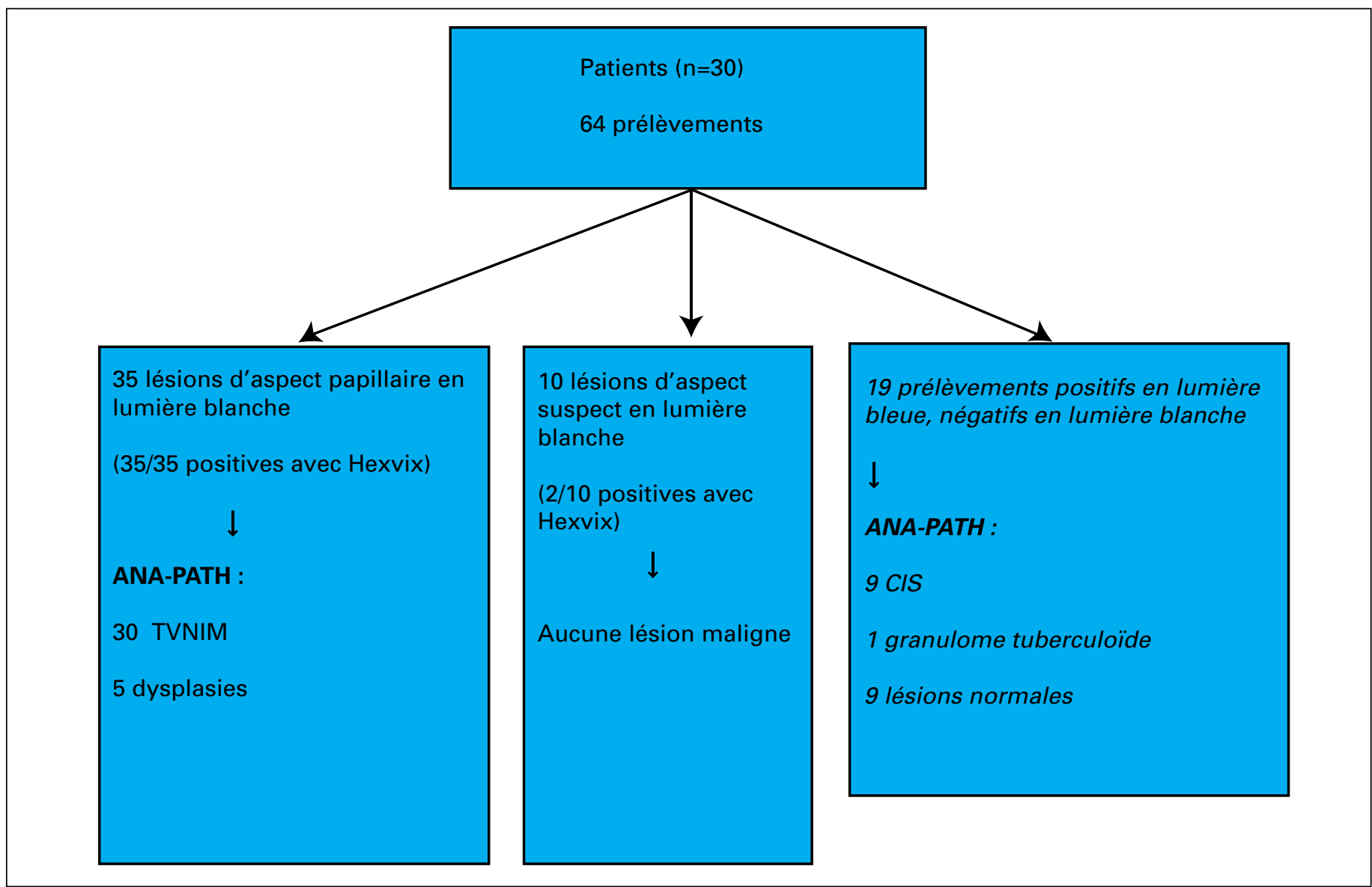

Fig. 3. Résultats de l'étude en fonction du type de lésion décelé.

2003, Jichlinski ${ }^{11}$ a signalé un taux de détection des lésions par HAL de $96 \%$, contre $73 \%$ par LB. La taille de notre échantillon peut expliquer cet écart de résultat, qui devra être confirmé par l'étude d'un échantillon plus important.

Le bénéfice est plus évident en ce qui concerne la détection de lésions planes (CIS, dysplasies). Dès 2004, Schmidbauer et coll ${ }^{13}$. ont publié les résultats de l'étude PCB301. Cette étude européenne multicentrique comparait la cystoscopie en fluorescence avec HAL à la cystoscopie en LB pour le diagnostic du CIS chez des patients à haut risque de cancer de la vessie. Il s'agissait d'une étude prospective contrôlée comparative. La vessie était cartographiée en lumière blanche, puis en lumière bleue. Sur 211 patients évaluables, 83 (39\%) avaient un CIS, dont 18 (22\%) un CIS seulement décelable par la fluorescence. Chez 62 des patients $(75 \%)$, le $\mathrm{CIS}$ a été détecté en LB et en lumière bleue; chez 2 des patients, il n'a été détecté qu'en LB ; et chez 1 seul des patients, il n'a été détecté que par la biopsie non guidée. Cette étude démontre les bénéfices de la fluorescence pour la détection du CIS, ce qui atteste notre résultat (100\% de CIS).

La principale lacune de l'HAL est son manque de spécificité, qui varie de $35 \%$ à $66 \%$, et qui semble même plus faible que celle de la cystoscopie standard dans plusieurs études. Le taux de faux positifs est en effet plus important en lumière bleue (environ $37 \%)^{8,9}$. La fréquence des faux positifs en lumière bleue observés dans notre étude était de $57,2 \%$, valeur qui reste élevée par rapport aux autres études. Elle était de $13 \%$ dans l'étude de Schmidbauer ${ }^{13}$, de $37 \%$ dans l'étude de Jocham ${ }^{8}$ et de $53 \%$ dans l'étude publiée par l'équipe espagnole d'Abascal Junquera ${ }^{14}$. Les faux positifs peuvent être attribuables à une inflammation ou à une thérapie intravésicale récente. Par ailleurs, I'observation de la muqueuse vésicale de façon tangentielle crée une impression de fausse fluorescence, particulièrement au col, au trigone et au niveau des diverticules vésicaux. Ces résultats soulignent le fait que cet examen dépend probablement de l'opérateur, sans oublier l'existence d'une courbe d'apprentissage liée à la lecture endoscopique en fluorescence.

D'autres hypothèses méritent cependant d'être discutées. Ainsi, la propension de certains opérateurs à effectuer des prélèvements invasifs dans les "zones d'aspect papillaire ou suspect » favorise probablement la fréquence des faux positifs. Enfin, ce taux de faux positifs rappelle les éventuels problèmes inhérents à la technique d'analyse histopathologique, problèmes qui sont courants dans notre série et les 
Sakel et al.

différentes séries publiées (mode d'inclusion des fragments, coupes étagées, épuisement du bloc).

Les résultats publiés signalent une diminution importante du taux de récidive des tumeurs de la vessie grâce à l'utilisation de l'HAL lors de la RTUV. La survie sans récidive tumorale passe d'un intervalle de $28 \%$ à $64 \%$ à un intervalle de $40 \%$ à 88 \% grâce à l'HAL. On dispose de données de suivi s'échelonnant sur une période allant jusqu'à huit ans. Cependant, on n'a pas observé de différences quant à la progression ou à la durée avant cystectomie. Aucun bénéfice particulier relatif à la survie n'a été démontré15-16.

\section{Conclusion}

L'utilisation de la fluorescence (Hexvix) lors de l'exploration vésicale avant la RTUV améliore sensiblement le diagnostic lié aux tumeurs n'infiltrant pas le muscle vésical, en particulier le diagnostic lié aux lésions de carcinome in situ. De plus, elle semble diminuer le risque de récidive tumorale. Cependant, selon les recommandations de l'AFU 2010, les indications de ce produit doivent être réservées à des situations cliniques particulières :

- lésions vésicales multifocales ;

- diamètre tumoral $>3 \mathrm{~cm}$;

- récidive tumorale précoce ;

- présence d'une cytologie de haut grade G3 ;

- surveillance des lésions vésicales à haut risque (T1G3 et $\mathrm{CIS}$ ).

Competing interests: Dr. Sakel, Dr. Mazdar and Dr. N'guibou all declare no competing financia or personal interests.

This paper has been peer-reviewed.

\section{Références}

1. Jemal A, Siegel R, Ward E, et al. Cancer statistics, 2009. Cancer J Clin 2009;59:225-49. http://dx.doi.org/10.3322/caac.20006

2. Ploeg $M$, Aben KK, Kiemeney LA. The present and future burden of urinary bladder cancer in the world. World J Urol 2009;27:289-93. http://dx.doi.org/10.1007/s00345-009-0383-3

3. Irani J, Bernardini S, Bonnal JL, et al. Tumeurs urotheliales. Recommandations du CCAFU. Prog Urol 2007;17:1065-98. http://dx.doi.org/10.1016/S1166-7087(07)74781-4

4. Herr HW. The value of a second transurethral resection in evaluating patients with bladder tumors. J Urol 1999;162:74-6. http://dx.doi.org/10.1097/00005392-199907000-00018

5. Sivalingam S, Probert JL, Schwaibold H. The role of repeat transurethral resection in the management of high-risk superficial transitional cell bladder cancer. BJU Int 2005;96:759-62. http://dx.doi.org/10.1111/i.1464-410X.2005.05710.x

6. Lee CS, Yoon CY, Wities JA. The past, present and future of cystoscopy: the fusion of cystoscopy and novel imaging technology. BJU Int 2008;102:1228-33. http://dx.doi.org/10.1111/i.1464-410X.2008.07964.x

7. Jichlinski P, Jacqmin D. Photodynamic diagnosis in non-muscle invasive blader cancer. Eur Urol Supp 2008:529.

8. Jocham D, Witjes $F$, Wagner $S$, et al. Improved detection and treatment of bladder cancer using hexaminolevulinate imaging: a prospective, phase III multicenter study. J Urol 2005;174:862-6. http://dx.doi.org/10.1097/01.ju.0000169257.19841.2a

9. Grossman HB, Gomella L, Fradet Y, et al.; PC B302/01 Study Group. A phase III, multicenter comparison of hexaminolevulinate fluorescence cystoscopy and white light cystoscopy for the detection of superficial papillary lesions in patients with bladder cancer. J Urol 2007; 178:62-7. http://dx.doi.org/10.1016/i.juro.2007.03.034

10. Ray ER, Chatterton K, Khan MS, et al. Hexylaminolaevulinate 'blue light' fluorescence cystoscopy in the investigation of clinically unconfirmed positive urine cytology. BJU Int 2009;103:1363-7. http://dx.doi.org/10.1111/i.1464-410X.2008.08238.x

11. Jichlinski P, Guillou L, Karlsen SJ, et al. Hexyl aminolevulinate fluorescence cystoscopy: new diagnostic tool for photodiagnosis of superficial bladder cancer-a multicenter study. J Urol 2003;170:226-9. http://dx.doi.org/10.1097/01.ju.0000060782.52358.04

12. Fradet $Y$, Grossman HB, Gomella L, et al. A comparison of hexaminolevulinate fluorescence cystoscopy and white light cystoscopy for the detection of carcinoma in situ in patients with bladder cancer: A phase III, multicenter study. J Urol 2007; 178:68-73. http://dx.doi.org/10.1016/i.juro.2007.03.028

13. Schmidbauer J, Witjes $F$, Schmeller N, et al. Improved detection of urothelial carcinoma in situ with hexaminolevulinate fluorescence cystoscopy. J Urol 2004;171:135-8. http://dx.doi.org/10.1097/01.ju.0000100480.70769.0e

14. Abascal Junquera JM, Hevia Suárez M, Abascal García JM, et al. Initial experience in the diagnosis and treatment of superficial bladder tumors with Hexvix®. Arch Esp Urol 2008;61:475-82. http://dx.doi.org/10.4321/S0004-06142008000400003

Correspondence: Dr. Adil Ait Sakel, Senvice d'urologie, Hôpital d’Auxerre, France ; sakel806@hotmail.com 